\title{
Comparison of the spontaneous activity, the linear response, and the stimulus-induced spike synchrony for three different integrate-and-fire models Benjamin Lindner* and Rafael D Vilela
}

\author{
Address: Biophysics Department, Max-Planck Institute for the Physics of Complex Systems, Dresden, Germany \\ Email: Benjamin Lindner* - benji@pks.mpg.de \\ * Corresponding author
}

from Eighteenth Annual Computational Neuroscience Meeting: CNS*2009

Berlin, Germany. 18-23 July 2009

Published: 13 July 2009

BMC Neuroscience 2009, I0(SuppI I):PI00 doi:I0.II86/I47|-2202-I0-SI-PI00

This abstract is available from: http://www.biomedcentral.com/I47I-2202/I0/SI/PI00

(c) 2009 Lindner and Vilela; licensee BioMed Central Ltd.

\section{Introduction}

Integrate-and-fire models are often employed in studies of the computational properties of neural systems (single neurons or neural networks). Different voltage-dependencies in the subthreshold dynamics have been used - from the very simple and analytically tractable perfect integrator to the more realistic but analytically more complicated leaky and quadratic IF models. Our comparison is based on parameter choices for the different models that make their firing rate and the $\mathrm{CV}$ of their interspike intervals equal. How different is the spontaneous activity of different models in terms of the ISI coefficient of variation or spike train power spectra? How do response and coherence functions of distinct IF models differ? And how strongly do the spike trains of two IF neurons correlate if they are driven by a common stimulus? How can we make the neurons input parameters comparable in the first place?

\section{Methods}

We study three different integrate-and-fire (IF) models, the perfect, leaky, and quadratic IF model driven by white Gaussian noise and present a systematic comparison of their spontaneous and driven firing statistics in terms of power spectra, susceptibilities, and coherence functions. To compare the parameter choices for the different models, we made their firing rates and the $\mathrm{CV}$ of their interspike intervals equal. We inspect the spontaneous power spectrum and the response functions for nine different fir- ing regimes. For selected parameter values we also look at the correlations induced in the spike trains of two neurons by a common stimulus.

\section{Results}

We prove that rate and CV uniquely determine the input parameters [1]. We find that power spectra and spontaneous activities are similar for all three models while the input-output and the correlation statistics depend on the specific voltage dependence of the model and on the firing regime (combination of rate and $\mathrm{CV}$ ) considered. We uncover relations between the strength of output correlations and the linear response of the respective model and demonstrate that depending on the firing regime either two leaky or two quadratic IF neurons correlate stronger. Furthermore, we show analytically that the output correlation of the spike counts of two perfect IF neurons is always equal to the input correlation.

\section{References}

I. Vilela RD, Lindner B: Are the input parameters of white noise driven integrate and fire neurons uniquely determined by rate and CV? J Theor Biol 2009, 257:90-99. 